\title{
Synthesis and Antimicrobial Evaluation of Some Novel 2-(Hydroxyimino)-dihydropyrimidine-5- carboxylic acids
}

\author{
R. A. SHASTRI
}

Post Graduate Department of Chemistry, S.B.E.S. College of Science, Aurangabad- 431001 (Maharashtra), India

shastriranjana@yahoo.com

Received 20 May 2019 / Accepted 3 July 2019

\begin{abstract}
A series of of 1,2,3,4-tetrahydro-2-(hydroxyimino)-6-methyl/1,6-dimethyl-4-phenyl pyrimidine-5-carboxylic acid (2a-o) were prepared by refluxing ethyl-1,2,3,4-tetrahydro-6methyl/1,6-dimethyl-2-oxo-4-phenylpyrimidine-5-carboxylate (1a-o) with hydroxylamine hydrochloride in the presence of sodium hydroxide, yielded (2a-o) in excellent yields. The advantages of this method is excellent yields, short reaction time, no side reaction and operational simplicity and ease product isolation The structure of all the synthesized compounds were characterized by FT-IR, ${ }^{1} \mathrm{H}$ NMR and MS data. The compounds 2a-o has been screened for their antimicrobial activity. All the compounds possesses significant to moderate antibacterial activity and promising antifungal activity.
\end{abstract}

Keywords: Dihydropyrimidinones, Hydroxylamine hydrochloride, Sodium hydroxide

\section{Introduction}

Dihydropyrimidinones have been subjected to a large number of different modifications in order to obtain derivatives having different biological properties. The (DHPMs) have attracted great attention recently in synthetic organic chemistry due to their applications in the field of drug research and pharmacological and therapeutic properties such as antibacterial $^{1}$, anti-inflammatory ${ }^{2}$, antiviral ${ }^{3}$, antitumor ${ }^{4}$, antimalarial agents ${ }^{5}$, hypnotics, anticonvulsant, antithyroid, antihistaminic agents, antibiotics ${ }^{6}$ and in addition, 4-aryldihydropyrimidines have emerged antihypertensive activity as well as behaving as calcium channel blockers $^{7,8}, \alpha$-antagonists and neuropeptide Y (NPY) antagonists ${ }^{9}$.

The versatile biological properties of DHPMs derivatives prompted us to synthesize oxime derivatives. In the present study, we report the synthesis of oxime derivatives by a new facile protocol. These compounds not only represent a useful series of derivatives of carbonyl compounds but also may be used as intermediates for the preparation of wide spectrum of organic compounds and numerous functional group transformations. 
Recently oximes and their derivatives have drawn attention in medicinal research because of their significant bioactivity.Oxime compounds are used as antidotes for nerve agents. A nerve agent inactivates acetylcholinesterase by phosphorylation. Oxime compounds can reactivate acetylcholinesterase by attaching to phosphorus, forming an oxime-phosphonate, which then splits away from the acetylcholinesterase molecule. Oxime nerve-agent antidotes are pralidoxime (also known as 2-PAM), obidoxime and methoxime are commonly used. A number of oximes have been used experimentally as anticonvulsants against electric shock.

In inorganic chemistry, oximes act as a versatile ligand. Moreover, oximes can be easily reduced to amines, which are further used in the manufacturing of dyes, plastics, synthetic fibres and pharmaceuticals. Oximes are used as anti-skinning agents in paint and blocking agents in the polymer industry.

Literature survey reveals that, no reports are available for oxime substitution in dihydropyrimidinones nucleus. The present attempt is to substitute carbonyl by oxime group in the molecular framework of DHPM to obtain new molecule with the intention that this group may display intensified bioactivity. In continuation of our earlier work, carried to develop convenient synthetic protocol for the synthesis of bioactive heterocyclic, herein we report the synthesis of new 1, 2,3,4-tetrahydro-2-(hydroxyimino)-6-methyl/1, 6-dimethyl -4phenylpyrimidine-5-carboxylic acid.

\section{Experimental}

Chemicals and solvents required were from Merck and SD fine. All melting points were determined in open capillaries in paraffin bath and are uncorrected. The progress of the reaction was monitored by thin layer chromatography. The products were characterized by spectral data. IR spectra were recorded on Perkin -Elmer FTIR spectrophotometer in $\mathrm{KBr}$ disc. ${ }^{1} \mathrm{H}$ NMR spectra were recorded on Bruker advance at $400 \mathrm{MHz}$ in $\mathrm{CDCl}_{3}$ as solvent and chemical shift values are recorded in ppm relative to tetramethylsilane as an internal standard.

General procedure for the synthesis of 1, 2, 3, 4-tetrahydro-2-(hydroxyimino)-6methyl/1, 6-dimethyl-4-Phenyl pyrimidine-5-carboxylic acid (2a-o)

To a stirred solution of ethyl-1,2,3,4-tetrahydro-6-methyl/ 1,6-dimethyl-2-oxo-4-phenylpyrimidine-5-carboxylate $(1 \mathrm{mmol})$ in ethanol $(10 \mathrm{~mL})$ hydroxylamine hydrochloride $(1.5$ $\mathrm{mmol}$ ) in $5 \mathrm{~mL}$ water and sodium hydroxide $(3 \mathrm{mmol})$ in $5 \mathrm{~mL}$ water were added and heated under reflux for $120 \mathrm{~min}$. The progress of the reaction was monitored by thin layer chromatography on Merck plates (silica gel 60F-254) using solvent $n$-hexane: ethyl acetate (9:1) and after completion of reaction mixture was allowed to cool and poured on ice cold water. The solid was filtered, washed with water and crystallized by aqueous ethanol and characterized by spectroscopic techniques. The yield and melting points are listed in Table 1.

\section{Spectral data}

\section{4-Phenyl-1,2,3,4-tetrahydro-2-(hydroxyimino)-6-methylpyrimidine-5-carboxylic $\operatorname{acid}(2 a)$}

IR $\left(\mathrm{KBr},\left(\mathrm{cm}^{-1}\right)\right): 3395(=\mathrm{NOH}), 1680(>\mathrm{C}=\mathrm{O}$ of acid $), 2425-3230(\mathrm{OH}$ of acid $), 2985(\mathrm{C}-\mathrm{H}$ aliph), 3119 (C-H aroma), 3224(N-H). ${ }^{1} \mathrm{H}$ NMR (CDCl3): $\delta$ (ppm): $1.29(\mathrm{~s}, 1 \mathrm{H}, \mathrm{N}-\mathrm{H}), 1.60$ $(\mathrm{s}, 1 \mathrm{H}, \mathrm{N}-\mathrm{H}), 2.35\left(\mathrm{~s}, 3 \mathrm{H}, \mathrm{CH}_{3}\right), 2.35(\mathrm{~s}, 1 \mathrm{H},=\mathrm{N}-\mathrm{OH}) 6.95(\mathrm{~d}, 1 \mathrm{H}, \mathrm{CH}), 7.15-7.95(\mathrm{~m}, 5 \mathrm{H}, \mathrm{Ar}-\mathrm{H})$, 8.49(S,1H,COOH) Es-MI. $246\left(\mathrm{M}^{+}\right) 100 \%$. 
4-(4-Chlorophenyl)-1,2,3,4-tetrahydro-2-(hydroxyimino)-6-methylpyrimidine-5 carboxylic acid (2e)

IR $\left(\mathrm{KBr},\left(\mathrm{cm}^{-1}\right)\right): 3390(=\mathrm{NOH}), 1675(>\mathrm{C}=\mathrm{O}$ of acid $), 2425-3250(\mathrm{OH}$ of acid $), 2985(\mathrm{C}-\mathrm{H}$ aliph), 3109 (C-H aroma), $3228(\mathrm{~N}-\mathrm{H}) .{ }^{1} \mathrm{H}$ NMR (CDCl3): $\delta(\mathrm{ppm}): 1.27$ (s, 1H, N-H), 1.60 (s, $1 \mathrm{H}, \mathrm{N}-\mathrm{H}), 2.35\left(\mathrm{~s}, 3 \mathrm{H}, \mathrm{CH}_{3}\right), 2.37(\mathrm{~s}, 1 \mathrm{H},=\mathrm{N}-\mathrm{OH}) 6.90(\mathrm{~d}, 1 \mathrm{H}, \mathrm{CH}), 7.05-7.90(\mathrm{~m}, 4 \mathrm{H}, \mathrm{Ar}-\mathrm{H})$, 8.49(s,1H,COOH) Es-MI. $281\left(\mathrm{M}^{+}\right) 100 \%$.

4-(4-Flulorophenyl)-1,2,3,4-tetrahydro-2-(hydroxyimino)-1,6-methylpyrimidine-5carboxylic acid (2i)

IR $\left(\mathrm{KBr},\left(\mathrm{cm}^{-1}\right)\right): 3392$ (=NOH), 1678(>C=O of acid), 2400-3200 (OH of acid), $2981(\mathrm{C}-\mathrm{H}$ aliph), 3109 (C-H aroma), $3224(\mathrm{~N}-\mathrm{H}) .{ }^{1} \mathrm{H}$ NMR (CDCl3): $\delta(\mathrm{ppm}): 1.26$ (s, 1H, N-H), 1.38 $\left(\mathrm{s}, 3 \mathrm{H}, \mathrm{N}-\mathrm{CH}_{3}\right), 2.30\left(\mathrm{~s}, 3 \mathrm{H}, \mathrm{CH}_{3}\right), 2.35(\mathrm{~s}, 1 \mathrm{H},=\mathrm{N}-\mathrm{OH}) 7.00(\mathrm{~d}, 1 \mathrm{H}, \mathrm{CH}), 7.05-7.90(\mathrm{~m}, 4 \mathrm{H}, \mathrm{Ar}-$ $\mathrm{H}), 8.48(\mathrm{~s}, 1 \mathrm{H}, \mathrm{COOH})$ Es-MI. $265\left(\mathrm{M}^{+}\right) 100 \%$.

4-(4-Methoxyphenyl)-1,2,3,4-tetrahydro-2-(hydroxyimino)-1,6-dimethylpyrimidine5-carboxylic acid(2g)

IR $\left(\mathrm{KBr},\left(\mathrm{cm}^{-1}\right)\right): 3395(=\mathrm{NOH}), 1678(>\mathrm{C}=\mathrm{O}$ of acid $), 2400-3200(\mathrm{OH}$ of acid $), 2981(\mathrm{C}-\mathrm{H}$ aliph), 3109 (C-H aroma), 3224(N-H). ${ }^{1} \mathrm{H}$ NMR (CDCl3): $\delta(\mathrm{ppm}): 1.16(\mathrm{~s}, 1 \mathrm{H}, \mathrm{N}-\mathrm{H})$, 1.20(s,3H, CH3), 1.71(s,1H,=N-OH) $2.38\left(\mathrm{~s}, 3 \mathrm{H}, \mathrm{OCH}_{3}\right) 5.65(\mathrm{~d}, 1 \mathrm{H}, \mathrm{CH}), 7.05-7.90(\mathrm{~m}, 4 \mathrm{H}$, Ar-H), 8.48(s,1H,COOH) Es-MI. $291\left(\mathrm{M}^{+}\right) 100 \%$.

4-(3-Bromophenyl)-1,2,3,4-tetrahydro-2-(hydroxyimino)-1,6-dimethylpyrimidine-5carboxylic acid (2l)

IR $\left(\mathrm{KBr},\left(\mathrm{cm}^{-1}\right)\right): 3390(=\mathrm{NOH}), 1676(>\mathrm{C}=\mathrm{O}$ of acid), 2400-3232 (OH of acid), 2985(C-H aliph), 3125 (C-H aroma), 3220(N-H). ${ }^{1} \mathrm{H}$ NMR (CDCl3): $\delta(\mathrm{ppm}): 1.16(\mathrm{~s}, 1 \mathrm{H}, \mathrm{N}-\mathrm{H})$, 1.20(s,3H, $\left.\mathrm{CH}_{3}\right), 1.71(\mathrm{~s}, 1 \mathrm{H},=\mathrm{N}-\mathrm{OH}), 5.65(\mathrm{~d}, 1 \mathrm{H}, \mathrm{CH}), 7.15-7.95(\mathrm{~m}, 4 \mathrm{H}, \mathrm{Ar}-\mathrm{H}), 8.50$ $(\mathrm{s}, 1 \mathrm{H}, \mathrm{COOH})$ Es-MI. $340\left(\mathrm{M}^{+}\right) 100 \%$.

\section{Results and Discussion}

In continuation of our research to develop methods for various transformations ${ }^{10}$, we herein report protocol for the synthesis of new1,2,3,4-tetrahydro-2-(hydroxyimino)-6-methyl-4substituted phenyl pyrimidine-5-carboxylic acid. The starting compounds DHPM (1a-o) required for this conversion are synthesized by treating urea/ $N$-methyl urea, substituted aromatic aldehyde and ethyl acetoacetate in the presence of PTSA in ethanol, by the reported procedure in literature ${ }^{11}$. Synthesized (1a-o) on reacting with hydroxylamine hydrochloride in the presence of sodium hydroxide, yielded (2a-o) Scheme 1. During oximation insitu ester group of dihydropyrimidinones is hydrolysed to acid. Compounds $2 \mathrm{a}-$ o were soluble in sodium bicarbonate solution and did not give 2, 4 dinitrophenyl hydrazine test indicating the absence of carbonyl group.

A structural evaluation of the new DHPM derivatives synthesized in this study was performed using spectroscopic techniques. The IR spectra of $2 \mathbf{e}$ showed normal stretching absorption band, indicating the existence of the $=\mathrm{NOH}\left(3390 \mathrm{~cm}^{-1}\right), \mathrm{N}-\mathrm{H}\left(3228 \mathrm{~cm}^{-1}\right)$, broad absorption from $2425-3250 \mathrm{~cm}^{-1}$ (OH of acid) and carbonyl $\left(1675 \mathrm{~cm}^{-1}\right)$ groups. ${ }^{1} \mathrm{H}$ NMR showed the presence of $\mathrm{N}_{1}-\mathrm{H}$ and $\mathrm{N}_{3}-\mathrm{H}$ proton as singlet at $\delta 1.20$ and at $\delta 1.60$ respectively, aromatic region showed the presence of protons in the region $87.00-7.90$. The acidic proton appears at $\delta 8.48$ indicating intramolecular hydrogen bonding between methyl group and acidic group. The ESI-MS of compound revealed the existence of their molecular ion peak, which is in accordance with the structure. 
<smiles>CC1=C(C(=O)O)C(c2ccccc2)NC(=O)N1</smiles>

1a-o<smiles>CC1=C(C(=O)O)C(c2ccc([Al])cc2)NC(=O)N1</smiles>

$2 \mathrm{a}-\mathrm{o}$

$\begin{array}{ll}\text { 1a-h } & \mathrm{R}_{1}=\mathrm{H} \\ \text { 1i-o } & \mathrm{R}_{1}=\mathrm{Me}\end{array} \quad \mathrm{R3}=\mathrm{OCH}_{2} \mathrm{CH}_{3}$

Scheme 1

Table 1. Synthesis of 1, 2, 3, 4-tetrahydro-2-(hydroxyimino)-6-methyl/1,6-dimethyl -4Phenyl pyrimidine-5-carboxylic acid (2a-o)

\begin{tabular}{ccccc}
\hline Entry & $\mathrm{R}_{1}$ & $\mathrm{R}_{2}$ & Yield\% & M.P. ${ }^{\circ} \mathrm{C}$ \\
\hline $\mathbf{2 a}$ & $\mathrm{H}$ & $\mathrm{H}$ & 80 & 140 \\
$\mathbf{2 b}$ & $\mathrm{H}$ & $4-\mathrm{F}$ & 85 & 142 \\
$\mathbf{2 c}$ & $\mathrm{H}$ & $4-\mathrm{OCH} \mathrm{H}_{3}$ & 89 & $164-66$ \\
$\mathbf{2 d}$ & $\mathrm{H}$ & $2-\mathrm{OH}$ & 84 & $174-76$ \\
$\mathbf{2 e}$ & $\mathrm{H}$ & $4-\mathrm{Cl}$ & 90 & $126-28$ \\
$\mathbf{2 f}$ & $\mathrm{H}$ & $3-\mathrm{NO}_{2}$ & 80 & $170-72$ \\
$\mathbf{2 g}$ & $\mathrm{H}$ & $3,4,5-\mathrm{Trimethoxy}$ & 80 & $176-78$ \\
$\mathbf{2 h}$ & $\mathrm{CH}$ & $\mathrm{H}$ & 85 & $168-70$ \\
$\mathbf{2 i}$ & $\mathrm{CH}_{3}$ & $4-\mathrm{F}$ & 90 & $170-71$ \\
$\mathbf{2 j}$ & $\mathrm{CH}_{3}$ & $4-\mathrm{OCH}$ & 85 & $148-50$ \\
$\mathbf{2 k}$ & $\mathrm{CH}_{3}$ & $2-\mathrm{Cl}$ & 78 & $172-174$ \\
$\mathbf{2 l}$ & $\mathrm{CH}_{3}$ & $3-\mathrm{NO} \mathrm{O}_{2}$ & 80 & $198-200$ \\
$\mathbf{2 m}$ & $\mathrm{CH}_{3}$ & $3,4,5-\mathrm{Trimethoxy}$ & 85 & $184-86$ \\
$\mathbf{2 n}$ & $\mathrm{CH}_{3}$ & 3-Br & 90 & $182-85$ \\
$\mathbf{2 0}$ & $\mathrm{CH}_{3}$ & $4-\mathrm{Br}$ & 92 & $122-24$ \\
\hline
\end{tabular}

\section{Antimicrobial evaluation}

The compounds 2a-o were screened for their antibacterial activity against Bacillus Subtilis and E.Coli using penicilline as reference standered by disc diffusion method ${ }^{12}$ and antifungal activity against Aspergillus niger and Pencillium Chrysogenum using Griseofulvin as reference standered by poison plate $\operatorname{method}^{13}$. Slandered antibacterial Penicillin and antifungal Griseofulvin were also screened under similar conditions for comparison. Results are presented in Table 2 .

The investigation of antibacterial screening result indicates that compounds $\mathbf{2 b}, \mathbf{2 c}, \mathbf{2 g}$, $\mathbf{2}, \mathbf{2 m}$ an $\mathbf{2} \mathbf{i}$ shows promising activity and compounds $\mathbf{2 a}, \mathbf{2 f}$ shows moderate activity whereas $\mathbf{2 d}$ shows low activity against E.Coli. Compounds $\mathbf{2 i}$ and $\mathbf{2 g}$ exhibit high activity and $\mathbf{2 b}, \mathbf{2 c}, \mathbf{2 l}$ possesses moderate activity and $\mathbf{2 a}, \mathbf{2 d}$ shows low activity against Bacillus Subtilis.

The investigation of antifungal data revealed that compounds $2 \mathbf{2 a}, \mathbf{2 b}, \mathbf{2 c}, \mathbf{2 d}, \mathbf{2 g}, \mathbf{2 i}, \mathbf{2 l}$ and $\mathbf{2 m}$ have inhibitory effect against Aspergillus niger whereas compounds $\mathbf{2 b}, \mathbf{2 c}, \mathbf{2 g}, \mathbf{2 i}$, $\mathbf{2 l}$ and $\mathbf{2 m}$ showed inhibitory effect and $\mathbf{2 a}, \mathbf{2 d}$ and $\mathbf{2 f}$ showed reduced growth against Pencillium Chrysogenum. 
Table 2. Antibacterial and antifungal activity for compounds (2a-o)

\begin{tabular}{cccccc}
\hline Compounds & $\begin{array}{c}\text { Bacillus } \\
\text { subtilis }\end{array}$ & $\begin{array}{c}\text { Escherichia } \\
\text { coli }\end{array}$ & Compounds & $\begin{array}{c}\text { Aspergillus } \\
\text { niger }\end{array}$ & $\begin{array}{c}\text { Penicillum } \\
\text { chrysogenum }\end{array}$ \\
\hline $\mathbf{2 a}$ & 12 & 10 & $2 \mathrm{a}$ & $-\mathrm{ve}$ & RG \\
$\mathbf{2 b}$ & 20 & 18 & $2 \mathrm{~b}$ & $-\mathrm{ve}$ & $-\mathrm{ve}$ \\
$\mathbf{2 c}$ & 19 & 16 & $2 \mathrm{c}$ & $-\mathrm{ve}$ & $-\mathrm{ve}$ \\
$\mathbf{2 d}$ & 10 & 08 & $2 \mathrm{~d}$ & $-\mathrm{ve}$ & RG \\
$\mathbf{2 f}$ & 09 & 11 & $2 \mathrm{e}$ & $+\mathrm{ve}$ & $-\mathrm{ve}$ \\
$\mathbf{2 g}$ & 22 & 18 & $2 \mathrm{f}$ & $\mathrm{RG}$ & RG \\
$\mathbf{2}$ & 24 & 15 & $2 \mathrm{~g}$ & $-\mathrm{ve}$ & $-\mathrm{ve}$ \\
$\mathbf{2}$ & 17 & 17 & $2 \mathrm{i}$ & $-\mathrm{ve}$ & $-\mathrm{ve}$ \\
$\mathbf{2}$ & 18 & 16 & $2 \mathrm{l}$ & $-\mathrm{ve}$ & $-\mathrm{ve}$ \\
$\mathbf{2 n}$ & Not tested & Not tested & $2 \mathrm{~m}$ & $-\mathrm{ve}$ & $-\mathrm{ve}$ \\
$\mathbf{2 0}$ & Not tested & Not tested & $2 \mathrm{n}$ & Not tested & Not tested \\
& & & $2 \mathrm{o}$ & Not tested & Not tested \\
Penicilline & 30 & 20 & Griseofulvin & $-\mathrm{ve}$ & s-ve \\
DMSO & $-\mathrm{ve}$ & $-\mathrm{ve}$ & DMSO & $+\mathrm{ve}$ & $+\mathrm{ve}$ \\
\hline
\end{tabular}

-ve No growth Antifungal activity, +ve Growth Antifungal activity absent, RG Reduced Growth

\section{Conclusion}

In conclusion, some novel compounds 4-(phenyl substituted)-1,2,3,4-tetrahydro-2(hydroxyimino)-6-methyl/1,6-dimethylpyrimidine-5-carboxylic acids have been synthesized. Both the functional groups oxime and acid have important applications in medicinal chemistry, the synthesized derivatives may act as good biological compounds.

\section{Acknowledgement}

Author thanks to SAIF, Chandigarh for providing IR and ${ }^{1} \mathrm{H}$ NMR spectral facilities. Author thanks to SAIF, Lucknow for providing Mass spectra. Author thanks to Dr.S. B.Jadhav Balbhim Arts, Science and Commerce College, Beed for help in antimicrobial activity.

\section{References}

1 Pansuriya A M, Savant M, Bhuva C V, Singh J and Naliapara Y T, Arkat USA J., 2009, 79-85.

2 Hatamjafari F and Nezhad F G, Oriental J Chem.., 2014, 30(1), 355-357; DOI:10.13005/ojc/300148

3 Hua K M and Le T N, Green Sustainable Chem., 2013, 3, 14-17; DOI:10.4236/gsc.2013.34A003

$4 \quad$ Nevagi R J and Narkhede H I, Scholars Research Library, 2014, 6(3), 135-139.

5 Hojati S F, Gholizadeh M, Haghdoust M and Shafiezadeh F, Bulletin Korean Chem Soc., 2010, 31(11), 3238-3239; DOI:10.5012/bkcs.2010.31.11.3238

6 Aslam M and Verma S, Int J Chemtech Res., 2012, 4, 109-111.

7 Nicholas A and Meanwell, J Med Chem., 2011, 54(8), 2529-2591; DOI:10.1021/jm1013693

8 Campbell K V, Leung A T and Sharp A H, Trends Neurosci., 1988, 11(10), 425-430; DOI:10.1016/0166-2236(88)90193-2

9 Reddy R, Reddy C V, Mahesh M, Raju P V and Reddy V V, Tetrahedron Lett., 2003, 44(44), 8173-8175; DOI:10.1016/j.tetlet.2003.09.030 
10 Shastri R A and Joshi P P, Chem Sci Trans., 2018, 7(1), 2278-3458; DOI:10.7598/cst2018

11 Shastri R A, Chem Sci Trans., 2018, 7(4), 708-714; DOI:10.7598/cst2018.1543

12 Hawkey P M and Lewis D A, Medical Bacteriology A Practicle Approach (Oxford University Press, Oxford, United Kingdom), 1994, 181.

13 Cruickshank R, A Practical of Medicinal Microbiology, Vol.2 (Churchill Livingstone, Edinburgh), 1975. 